\title{
REMARKS ON EMBEDDABLE SEMIGROUPS IN GROUPS AND A GENERALIZATION OF SOME CUTHBERT'S RESULTS
}

\author{
KHALID LATRACH and ABDELKADER DEHICI
}

Received 5 February 2001 and in revised form 27 July 2001

\begin{abstract}
Let $(U(t))_{t \geq 0}$ be a $C_{0}$-semigroup of bounded linear operators on a Banach space $X$. In this paper, we establish that if, for some $t_{0}>0, U\left(t_{0}\right)$ is a Fredholm (resp., semiFredholm) operator, then $(U(t))_{t \geq 0}$ is a Fredholm (resp., semi-Fredholm) semigroup. Moreover, we give a necessary and sufficient condition guaranteeing that $(U(t))_{t \geq 0}$ can be embedded in a $C_{0}$-group on $X$. Also we study semigroups which are near the identity in the sense that there exists $t_{0}>0$ such that $U\left(t_{0}\right)-I \in \mathscr{F}(X)$, where $\mathscr{f}(X)$ is an arbitrary closed two-sided ideal contained in the set of Fredholm perturbations. We close this paper by discussing the case where $\mathscr{f}(X)$ is replaced by some subsets of the set of polynomially compact perturbations.
\end{abstract}

2000 Mathematics Subject Classification: 47A53, 47A55, 47D03.

1. Introduction. Let $X$ be a Banach space over the complex field and let $\mathscr{L}(X)$ denote the Banach algebra of bounded linear operators on $X$. The subset of all compact operators of $\mathscr{L}(X)$ is designated by $\mathscr{L}(X)$. For $A \in \mathscr{L}(X)$, we let $\sigma(A), \rho(A), R(\lambda, A), N(A)$, and $R(A)$ denote the spectrum, the resolvent set, the resolvent operator, the null space, and the range of $A$, respectively. The nullity of $A, \alpha(A)$, is defined as the dimension $N(A)$ and the deficiency of $A$, $\beta(A)$, is defined as the codimension of $R(A)$ in $X$.

Write

$$
\begin{aligned}
& \Phi_{+}(X)=\{A \in \mathscr{L}(X): \alpha(A)<\infty, R(A) \text { is closed in } X\}, \\
& \Phi_{-}(X)=\{A \in \mathscr{L}(X): \beta(A)<\infty(\text { then } R(A) \text { is closed in } X)\} .
\end{aligned}
$$

By $\Phi_{ \pm}(X):=\Phi_{+}(X) \cup \Phi_{-}(X)$ we denote the set of semi-Fredholm operators in $\mathscr{L}(X)$, while $\Phi(X):=\Phi_{+}(X) \cap \Phi_{-}(X)$ is the set of Fredholm operators in $\mathscr{L}(X)$. If $A \in \Phi_{ \pm}(X)$, the number $i(A)=\alpha(A)-\beta(A)$, a finite or infinite integer is the index of $A$. Let $X^{*}$ denotes the dual space of $X$ and $A^{*}$ the dual operator of $A$.

Let $(U(t))_{t \geq 0}$ be a $C_{0}$-semigroup of bounded linear operators on $X$. We say that $(U(t))_{t \geq 0}$ is a Fredholm (resp., semi-Fredholm) semigroup if $U(t)$ is in $\Phi(X)$ (resp., $\left.\Phi_{ \pm}(X)\right)$ for all $t>0$. 
In [7, Theorem 16.3.6], it is proved that a $C_{0}$-semigroup of bounded linear operators $(U(t))_{t \geq 0}$ can be embedded in a $C_{0}$-group if and only if there exists $t_{0}>0$ such that $0 \in \rho\left(U\left(t_{0}\right)\right)$. The main goal of Section 2 is to give a generalization of this result to Fredholm semigroup. Our approach consists in relaxing the requirement there exists $t_{0}>0$ such that $0 \in \rho\left(U\left(t_{0}\right)\right)$ and replacing it by the weaker one there exists $t_{0}>0$ such that $U\left(t_{0}\right) \in \Phi(X)$. In fact, we prove under this hypothesis that $(U(t))_{t \geq 0}$ is a Fredholm semigroup, that is, $U(t) \in \Phi(X)$ for all $t \geq 0$. In particular, we show that if there exists $t_{0}>0$ such that $U\left(t_{0}\right) \in \Phi_{ \pm}(X)$, then $(U(t))_{t \geq 0}$ is a semi-Fredholm semigroup, that is, $U(t) \in \Phi_{ \pm}(X)$ for all $t \geq 0$.

In Section 3, we extend some results owing to Cuthbert [2] which deal with $C_{0}$-semigroups having the property of being near the identity, in the sense that, for some value of $t, U(t)-I \in \mathscr{K}(X)$. We show that Cuthbert's results remain valid if, for some $t_{0}>0, U\left(t_{0}\right)-I \in \mathscr{F}(X)$ where $\mathscr{g}(X)$ is an arbitrary closed twosided ideal of $\mathscr{L}(X)$ contained in the ideal of Fredholm perturbations $\mathscr{F}(X)$. In the last section, some generalizations of the results obtained in Section 3 to polynomially compact perturbations are also given.

2. Embeddable $C_{0}$-semigroups in $C_{0}$-groups. Let $X$ be a Banach space and let $(U(t))_{t \geq 0}$ be a $C_{0}$-semigroup of bounded linear operators on $X$.

THEOREM 2.1. A $C_{0}$-semigroup $(U(t))_{t \geq 0}$ can be embedded in a $C_{0}$-group on $X$ if and only if there exists $t_{0}>0$ such that $U\left(t_{0}\right) \in \Phi(X)$.

To prove Theorem 2.1, the following proposition is required.

Proposition 2.2. Let $t_{0}>0$ and let $(U(t))_{t \geq 0}$ be a $C_{0}$-semigroup on $X$.

(i) If $U\left(t_{0}\right) \in \Phi_{+}(X)$, then $U(t) \in \Phi_{+}(X)$ and $\alpha(U(t))=0$ for all $t \geq 0$.

(ii) If $U\left(t_{0}\right) \in \Phi_{-}(X)$, then $U(t) \in \Phi_{-}(X)$ and $\beta(U(t))=0$ for all $t \geq 0$.

(iii) If $U\left(t_{0}\right) \in \Phi(X)$, then $U(t) \in \Phi(X)$ and $i(U(t))=0$ for all $t \geq 0$.

Obviously, Proposition 2.2 shows that if, for some $t_{0}>0, U\left(t_{0}\right) \in \Phi_{ \pm}(X)$, then $(U(t))_{t \geq 0}$ is a semi-Fredholm semigroup. In the case where $U\left(t_{0}\right) \in \Phi(X)$, $(U(t))_{t \geq 0}$ is a Fredholm semigroup and $i(U(t))=0$ for all $t \geq 0$.

Proof of Proposition 2.2. (i) We first show that $U\left(t_{0}\right)$ is injective. Since $\alpha\left(U\left(t_{0}\right)\right)<\infty$, then 0 is an eigenvalue with finite multiplicity of $U\left(t_{0}\right)$. Let $x \neq 0$ be an eigenvector associated to 0 . Putting $t_{1}=t_{0} / 2$, then $U\left(t_{0}\right) x=$ $U\left(t_{1}\right) U\left(t_{1}\right) x=0$, hence 0 is an eigenvalue of $U\left(t_{1}\right)$. Proceeding by induction, we define a sequence $\left(t_{n}\right)_{n \in \mathbb{N}}$ with $t_{n} \rightarrow 0$ as $n \rightarrow \infty$ such that 0 is an eigenvalue of $U\left(t_{n}\right), \forall n \in \mathbb{N}$. For $n \geq 0$, we define the sets

$$
\Lambda_{n}=N\left(U\left(t_{n}\right)\right) \bigcap\{x \in X:\|x\|=1\} .
$$

Clearly, the inclusion $N(U(s)) \subseteq N(U(t))$, for $s \leq t$, and the compactness of $\Lambda_{0}$ imply that $\left(\Lambda_{n}\right)_{n}$ is a decreasing sequence (in the sense of the inclusion) of 
nonempty compact subsets of $X$. Thus $\bigcap_{n=0}^{\infty} \Lambda_{n} \neq \varnothing$. If $x \in \bigcap_{n=0}^{\infty} \Lambda_{n}$, then

$$
\left\|U\left(t_{n}\right) x-x\right\|=\|x\|=1 \quad \forall n \geq 1 .
$$

Since $t_{n} \rightarrow 0$ as $n \rightarrow \infty$, (2.2) contradicts the strong continuity of $(U(t))_{t \geq 0 \text {. }}$ This shows that $N\left(U\left(t_{0}\right)\right)=\{0\}$, that is, $\alpha\left(U\left(t_{0}\right)\right)=0$.

Let $0 \leq t \leq t_{0}$. The inclusion $N(U(t)) \subseteq N\left(U\left(t_{0}\right)\right)$ implies that $\alpha(U(t))=0$. Assume now that $t>t_{0}$ and $x \in N(U(t))$, then there exists an integer $n$ such that $n t_{0}>t$ and therefore $U\left(n t_{0}\right) x=U\left(n t_{0}-t\right) U(t) x=0$. Hence, we have $x=0$ and consequently $N(U(t))=\{0\}$ for all $t>t_{0}$ which ends the proof of (i).

(ii) To prove this item, we will proceed by duality. Let $\left(U^{*}(t)\right)_{t \geq 0}$ be the dual semigroup of $(U(t))_{t \geq 0}$. Since $\beta(U(t))=\alpha\left(U^{*}(t)\right)$, then it suffices to show that $\alpha\left(U^{*}(t)\right)=0$ for all $t \geq 0$. By hypothesis, we have $\alpha\left(U^{*}\left(t_{0}\right)\right)<\infty$. Let $x^{*}$ be an element of $N\left(U^{*}\left(t_{0}\right)\right)$. Arguing as above, we construct a sequence $\left(t_{n}\right)_{n \in \mathbb{N}}$ with $t_{n} \rightarrow 0$ as $n \rightarrow \infty$ such that 0 is an eigenvalue of $U^{*}\left(t_{n}\right)$, for all $n \in \mathbb{N}$ a decreasing sequence

$$
\Sigma_{n}=N\left(U^{*}\left(t_{n}\right)\right) \bigcap\left\{x^{*} \in X^{*}:\left\|x^{*}\right\|=1\right\}
$$

of nonempty compact subsets of $X^{*}$. We infer that $\bigcap_{n=0}^{\infty} \Sigma_{n} \neq \varnothing$. Let $x^{*} \in$ $\bigcap_{n=0}^{\infty} \Sigma_{n}$, then for all $n \in \mathbb{N}$

$$
\left|\left\langle U^{*}\left(t_{n}\right) x^{*}-x^{*}, x\right\rangle\right|=\left|\left\langle x^{*}, x\right\rangle\right| \quad \forall x \in X .
$$

Using the fact that $\left(U^{*}(t)\right)_{t \geq 0}$ is continuous in the weak* topology at $t=0$, we conclude that

$$
\lim _{t \rightarrow 0}\left|\left\langle U^{*}\left(t_{n}\right) x^{*}-x^{*}, x\right\rangle\right|=0 \quad \forall x \in X .
$$

Combining (2.4) and (2.5), we obtain $\left\langle x^{*}, x\right\rangle=0$ for all $x \in X$. This shows that $x^{*}=0$ and therefore $\alpha\left(U^{*}\left(t_{0}\right)\right)=0$. Arguing as above, we show that $\alpha\left(U^{*}(t)\right)=0$ for all $t \geq 0$.

(iii) This follows from (i) and (ii).

To complete the proof of (i) it suffices to show that $R(U(t))$ is closed in $X$ for all $t \geq 0$. Assume that $U\left(t_{0}\right) \in \Phi_{+}(X)$, then $\alpha\left(U\left(t_{0}\right)\right)<\infty$ and $\beta\left(U\left(t_{0}\right)\right)=\infty$ (if $\beta\left(U\left(t_{0}\right)\right)<\infty$ the proof is contained in (ii) see below). Let $U^{*}\left(t_{o}\right)$ be the dual operator of $U\left(t_{0}\right)$. Obviously, $U^{*}\left(t_{0}\right) \in \Phi_{-}(X)$ and consequently $\beta\left(U^{*}\left(t_{0}\right)\right)<$ $\infty$. Hence $\beta\left(U^{*}(t)\right)<\infty$ for all $t \geq 0$. Now applying Kato's lemma [8, Lemma 332] we infer that $R\left(U^{*}(t)\right)$ is closed in $X^{*}$ for all $t \geq 0$. This together with the closed graph theorem of Banach [15, page 205] implies that $R(U(t))$ is closed in $X$ for all $t \geq 0$.

Assume now that $U\left(t_{0}\right) \in \Phi_{-}(X)$, then $\beta\left(U\left(t_{0}\right)\right)<\infty$ and $\alpha\left(U\left(t_{0}\right)\right)=\infty$ (if $\alpha\left(U\left(t_{0}\right)\right)<\infty$ the proof is contained in (i)). It follows from the first part of the statement (ii) that $\beta(U(t))<\infty$ for all $t \geq 0$. Again using Kato's lemma 
[8, Lemma 332] we see that $R(U(t))$ is closed in $X$ for all $t \geq 0$ which completes the proof of (ii).

Now if $U\left(t_{0}\right) \in \Phi(X)$, then $\alpha\left(U\left(t_{0}\right)\right)<\infty$ and $\beta\left(U\left(t_{0}\right)\right)<\infty$. It follows from the discussion above that $R(U(t))$ is closed in $X$ for all $t \geq 0$. This ends the proof of Proposition 2.2.

Proof OF THeOrem 2.1. The proof follows immediately from Proposition 2.2 and [7, Theorem 16.3.6].

3. An extension of some results by Cuthbert. Throughout this section $X$ denotes a Banach space and $(U(t))_{t \geq 0}$ designates a strongly continuous semigroup with infinitesimal generator $A$.

As mentioned in the introduction, this section is motivated by Cuthbert's work [2] dealing with $C_{0}$-semigroups which have the property of being near the identity, in the sense that, for some positive value of $t>0, U(t)-I \in \mathscr{K}(X)$. We discuss the possibility of extending Cuthbert's results to other operator ideals of $\mathscr{L}(X)$. To this purpose, we introduce the concept of Fredholm perturbations (see $[1,4,12])$.

DEFINITION 3.1. We say that an operator $F \in \mathscr{L}(X)$ is a Fredholm perturbation if $A+F \in \Phi(X)$ whenever $A \in \Phi(X)$. The operator $F$ is called an upper (resp., lower) semi-Fredholm perturbation if $F+A \in \Phi_{+}(X)$ (resp., $F+A \in$ $\Phi_{-}(X)$ ) whenever $A \in \Phi_{+}(X)$ (resp., $A \in \Phi_{-}(X)$ ).

The sets of Fredholm, upper semi-Fredholm, and lower semi-Fredholm perturbations are denoted by $\mathscr{F}_{(}(X), \mathscr{F}_{+}(X)$, and $\mathscr{F}_{-}(X)$, respectively. These sets of operators were introduced and investigated in [4] (see also [12]). In particular, it is proved that $\mathscr{F}_{+}(X)$ and $\mathscr{F}_{F}(X)$ are closed two-sided ideals of $\mathscr{L}(X)$ while $\mathscr{F}_{-}(X)$ is a closed subset of $\mathscr{L}(X)$.

Our main objective here is to show that Cuthbert's results remain valid if we replace $\mathscr{K}(X)$ by any closed two-sided ideal contained in $\mathscr{F}(X)$.

In the following, $\mathscr{S}(X)$ denotes an arbitrary nonzero closed two-sided ideal of $\mathscr{L}(X)$ satisfying

$$
\mathscr{L}(X) \subseteq \mathscr{F}(X) .
$$

REMARK 3.2. (1) It is worth noticing that, in general, the structure ideal of $\mathscr{L}(X)$ is extremely complicated. Most of the results on ideal structure deal with the well-known closed ideals which have arisen from applied work with operators. We can quote, for example, compact operators, weakly compact operators, strictly singular operators, strictly cosingular operators, upper semi-Fredholm perturbations, and Fredholm perturbations. In general, we have

$$
\begin{aligned}
& \mathscr{K}(X) \subseteq \mathscr{Y}(X) \subseteq \mathscr{F}_{+}(X) \subseteq \mathscr{F}(X), \\
& \mathscr{K}(X) \subseteq C \mathscr{Y}(X) \subseteq \mathscr{F}_{-}(X) \subseteq \mathscr{F}(X),
\end{aligned}
$$


where $\mathscr{S}(X)$ and $C \mathscr{S}(X)$ denote, respectively, the ideals of $\mathscr{L}(X)$ consisting of strictly singular and strictly cosingular operators on $X$. The inclusion $\mathscr{S}(X) \subseteq$ $\mathscr{F}_{+}(X)$ is due to Kato (cf. [8]) while $C \mathscr{S}(X) \subseteq \mathscr{F}_{-}(X)$ was proved by Vladimirskiı [13].

(2) If $X$ is isomorphic to an $L_{p}$ space with $1 \leq p \leq \infty$ or to $C(\Xi)$ where $\Xi$ is a compact Hausdorff space, then we have

$$
\mathscr{K}(X) \subseteq \mathscr{Y}(X)=\mathscr{F}_{+}(X)=C \mathscr{S}(X)=\mathscr{F}_{-}(X)=\mathscr{F}(X)
$$

(cf. [9, equations (2.9) and (2.10)]).

A Banach space $X$ is said to be an $h$-space if each closed infinite-dimensional subspace of $X$ contains a complemented subspace isomorphic to $X$ [14]. Any Banach space isomorphic to an $h$-space; $c, c_{0}$ and $l_{p}(1 \leq p<\infty)$ are $h$-spaces. In [14, Theorem 6.2], Whitley proved that, if $X$ is an $h$-space, then $\mathscr{Y}(X)$ is the greatest proper ideal of $\mathscr{L}(X)$. This, together with (3.2), implies that

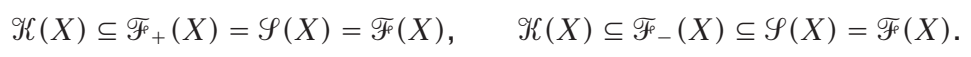

We denote by 0 the set

$$
\mathcal{O}=\{t>0 \text { such that } U(t)-I \in \mathscr{E}(X)\}
$$

It should be noted that for a given $C_{0}$-semigroup, the set 0 can be empty.

REMARK 3.3. Note that, under assumption (3.1), if $0 \neq \varnothing$, then the $C_{0}$ semigroup $(U(t))_{t \geq 0}$ can be embedded in a $C_{0}$-group on $X$. (It suffices to write $U\left(t_{0}\right)=I+\left[U\left(t_{0}\right)-I\right]$ for some $t_{o} \in \mathcal{O}$ and to apply Theorem 2.1.) This statement improves [2, Theorem 1].

Observe that the relation

$$
(U(t)-I)(U(s)-I)=(U(t+s)-I)-(U(s)-I)-(U(t)-I)
$$

implies that

$$
s \in \mathbb{O}, t \in \mathcal{O} \Longrightarrow s+t \in \mathcal{O}, \quad s \in \mathcal{O}, t \notin \mathbb{O} \Longrightarrow s+t \notin \mathcal{O} \text {. }
$$

It follows from these relations that $\mathrm{O}$ is the intersection of an additive subgroup of real number with the positive real line. Therefore, $O$ may be in one of the following forms:

(i) $0=] 0, \infty[$;

(ii) $0=\{n x$, for some $x>0$; and $n=1,2, \ldots\}$;

(iii) $\mathcal{O}$ is a dense subset of $] 0, \infty[$ with empty interior. 
The following examples taken from [2] show that all the three types of sets may occur, the above classification of 0 -sets is not empty; and sets of type (ii) can arise from semigroups having bounded or unbounded infinitesimal generators.

EXAMPLES 3.4. Take $X=l_{1}$, the Banach space of absolutely convergent sequences. As mentioned above (see Remark 3.2(1)), $\mathscr{K}(X)$ is the sole closed twosided proper ideal of $\mathscr{L}(X)$, that is, $\mathscr{K}(X)=\mathscr{F}(X)$.

(1) Let $(U(t))_{t \geq 0}$ be the $C_{0}$-semigroup given by $U(t)=I$ for all $t \geq 0$. Clearly, for all $t>0, U(t)-I \in \mathscr{K}(X)$. Accordingly, $\mathcal{O}=] 0, \infty[$ and $A=0$.

(2) (a) Assume that $U(t)=\operatorname{diag}\left\{e^{i t}, e^{-i t}, e^{i t}, e^{-i t}, \ldots\right\}$ for all $t \geq 0$. In this case, we have $\mathcal{O}=\{2 n \pi, n=1,2,3, \ldots\}$ and $A=\operatorname{diag}\{i,-i, i,-i, \ldots\}$, the infinitesimal generator of $(U(t))_{t \geq 0}$, is bounded.

(b) Suppose now that $U(t)=\operatorname{diag}\left\{e^{i t}, e^{2 i t}, e^{3 i t}, e^{4 i t}, \ldots\right\}$ for all $t \geq 0$. Here, we have also $O=\{2 n \pi, n=1,2,3, \ldots\}$ but $A=\operatorname{diag}\{i, 2 i, 3 i$, $4 i, \ldots\}$, the infinitesimal generator of $(U(t))_{t \geq 0}$, is unbounded.

(3) The $C_{0}$-semigroup $(U(t))_{t \geq 0}$ with $U(t)=\operatorname{diag}\left\{e^{i t}, e^{2 ! i t}, e^{3 ! i t}, \ldots, e^{n ! i t}, \ldots\right\}$ provides an example of 0 -set of type (iii).

In the next theorem, we derive some relationships between the type of 0 -sets and the structure of the semigroup. In particular, we show that 0 has the first form if and only if $A$ is a Fredholm perturbation. If $O$ takes the third form, then $A$ is necessarily unbounded.

THEOREM 3.5. Assume that condition (3.1) is satisfied. Then the following statements are equivalent:

(i) $\mathrm{O}=] 0,+\infty[$;

(ii) $A$ is a Fredholm perturbation;

(iii) $\lambda R(\lambda, A)-I$ is a Fredholm perturbation for some (in fact for all) $\lambda>\omega$.

This result extends [2, Theorem 2] to large classes of operators which contain properly the set of compact operators.

Proof OF Theorem 3.5. (i) $\Rightarrow$ (ii). The first step in the proof of this implication consists in showing that (i) implies that $A$ is bounded. The proof of this implication is similar to that of [2, Theorem 2]. Details are omitted.

Next, since $A$ is bounded, then $U(t)$ is uniformly continuous for $t \geq 0$ (see [7]). Hence, for all $\varepsilon>0$ there exists $\delta>0$ such that

$$
\|U(t)-I\|<\varepsilon \text { for } t<\delta
$$

Accordingly, for any $t<\delta$, we have

$$
\left\|\frac{1}{t} \int_{0}^{t} U(s) d s-I\right\|=\left\|\frac{1}{t} \int_{0}^{t}(U(s)-I) d s\right\| \leq \frac{1}{t} \int_{0}^{t}\|U(s)-I\| d s<\varepsilon .
$$


Hence, for $\varepsilon$ small enough, $\int_{0}^{t} U(s) d s$ is invertible for all $t<\delta$. Moreover, using the identity

$$
U(t)-I=A \int_{0}^{t} U(s) d s
$$

together with the fact that $A$ and $U(t)$ commute, we infer that

$$
A=\left[\int_{0}^{t} U(s) d s\right]^{-1}(U(t)-I) .
$$

Since $\mathscr{E}(X)$ is an ideal, we infer that $A \in \mathscr{F}(X)$.

(ii) $\Rightarrow$ (i). Assume that $A \in \mathscr{F}(X)$. Using again identity (3.10) and the ideal structure of $\mathscr{f}(X)$ we see that $U(t)-I \in \mathscr{E}(X)$ for all $t \geq 0$.

(ii) $\Rightarrow$ (iii). This follows from the identity $\lambda R(\lambda, A)-I=A R(\lambda, A)$ and the ideal structure of $\mathscr{g}(X)$.

(iii) $\Rightarrow$ (ii). Assume that $\lambda R(\lambda, A)-I \in \mathscr{F}(X)$ for all $\lambda>\omega$. Note that the identity $\lambda R(\lambda, A)-I=A R(\lambda, A)$ and (3.11) lead to

$$
R(\lambda, A)(U(t)-I)=(\lambda R(\lambda, A)-I) \int_{0}^{t} U(s) d s \quad \forall t \geq 0 .
$$

Writing (3.12) in the form

$$
\begin{gathered}
\lambda R(\lambda, A)(U(t)-I)-(U(t)-I)+(U(t)-I) \\
=\lambda(\lambda R(\lambda, A)-I) \int_{0}^{t} U(s) d s \quad \forall t \geq 0,
\end{gathered}
$$

we infer that

$$
U(t)-I=(\lambda R(\lambda, A)-I)\left[U(t)-I+\lambda \int_{0}^{t} U(s) d s\right]
$$

Next, using the fact that $\left[U(t)-I+\lambda \int_{0}^{t} U(s) d s\right] \in \mathscr{L}(X)$, we get that $U(t)-I \in$ $\mathscr{F}(X)$ for all $t \geq 0$, that is, $O=] 0, \infty[$. This achieves the proof.

The next result asserts that if the $\mathbf{O}$-set is in the form (iii), then the infinitesimal generator of $(U(t))_{t \geq 0}$ is necessarily unbounded. It generalizes [2, Theorem 3].

Proposition 3.6. Assume that condition (3.1) holds true. If 0 is a dense subset of $] 0, \infty[$ with no interior points, then $A$ is unbounded.

Proof. Assume, for contradiction, that $A$ is bounded. Then, proceeding as in the proof of the implication (i) $\Rightarrow$ (ii) in Theorem 3.5 we see that if $t<\delta$ and $t \in \mathcal{O}$, then $A \in \mathscr{g}(X)$. So, by Theorem 3.5, we get $\mathrm{O}=$ ] $0, \infty$ [. This contradicts the hypothesis. 
REMARK 3.7. (1) Notice that if $\mathscr{f}(X)$ is a nonzero closed two-sided ideal of $\mathscr{L}(X)$ satisfying (3.1), then it follows from [4, Proposition 4, page 70] that

$$
\overline{\mathscr{F}_{0}(X)} \subseteq \mathscr{g}(X) \subseteq \mathscr{F}(X),
$$

where $\mathscr{F}_{0}(X)$ stands for the ideal of finite rank operators on $X$. This shows that $\overline{\mathscr{F}_{0}}(X)$ is the minimal ideal (in the sense of the inclusion) in $\mathscr{L}(X)$ for which the results of this section are valid. Evidently, if $X$ has the approximation property, then we have $\overline{\mathscr{F}_{0}(X)}=\mathscr{K}(X)$.

(2) Even though the description of the ideal structure of $\mathscr{L}(X)$ is a complex task, there exist some Banach spaces $X$ for which $\mathscr{L}(X)$ has only one proper nonzero closed two-sided ideal. The first result in this direction was established by Calkin (cf. [4]). He proved that if $X$ is a separable Hilbert space, then $\mathscr{K}(X)$ is the unique proper nonzero closed two-sided ideal of $\mathscr{L}(X)$. An extension of this result was obtained by Gohberg et al. [4]. They proved the same result for $X=l_{p}, 1 \leq p<\infty$, and $X=c_{0}$. In [6], Herman establishes the same result for a large class of Banach spaces, namely Banach spaces which have perfectly homogeneous block bases and satisfy $(+)$ (for the definition and the meaning of the symbol (+) we refer to [6]). (Evidently, the spaces $l_{p}, 1 \leq p<\infty$, and $c_{0}$ belong to this class.) Thus, if $X$ has perfectly homogeneous block bases which satisfy $(+)$, then

$$
\mathscr{K}(X)=\mathscr{F}_{+}(X)=\mathscr{F}_{-}(X)=\mathscr{F}_{(X)} .
$$

Consequently, for this class of spaces the results of this section use the ideal of compact operators and coincide with those obtained in [2]. Hence, for such spaces the Cuthbert results are optimal.

4. Further extensions. Let $X$ be a Banach space. An operator $R \in \mathscr{L}(X)$ is called a Riesz operator if $\lambda-R \in \Phi(X)$ for all scalars $\lambda \neq 0$. Let $\mathscr{R}(X)$ denote the class of all Riesz operators. For further discussions concerning this family of operators, we refer to [1, 12] and the references therein. For our purpose, we recall that Riesz operators satisfy the Riesz-Schauder theory of compact operators, $\mathscr{R}(X)$ is not an ideal of $\mathscr{L}(X)$ [1], and $\mathscr{F}(X)$ is the largest ideal contained in $\mathscr{R}(X)$ [12]. Hence the sets $\mathscr{K}(X), \mathscr{Y}(X), C \mathscr{Y}(X), \mathscr{F}_{+}(X)$, and $\mathscr{F}_{-}(X)$ are also contained in $\mathscr{R}(X)$.

Let $A \in \mathscr{L}(X)$. The Fredholm region of $A$ is defined as $\{\lambda \in \mathbb{C} ; \lambda-A \in \Phi(X)\}$ and denoted by $\Phi_{A}$. Next, let $\Phi_{A}^{0}:=\left\{\lambda \in \Phi_{A}: i(\lambda-A)=0\right\}$ and define the set

$$
\sigma_{b}(A):=\mathbb{C} \backslash \rho_{b}(A),
$$

where

$$
\rho_{b}(A):=\left\{\lambda \in \Phi_{A}^{0} \text { such that all scalars near } \lambda \text { are in } \rho(A)\right\} .
$$

Following $[5,11], \sigma_{b}(\cdot)$ is called the Browder essential spectrum. 
We say that an operator $F \in \mathscr{L}(X)$ is polynomially compact (see [3]) if there is a nonzero complex polynomial $p(z)$ such that the operator $p(F)$ is compact. We designate by $P \mathscr{K}(X)$ the set of polynomially compact operators on $X$. Let $F \in P \mathscr{K}(X)$, the nonzero polynomial $p(z)$ of least degree and leading coefficient 1 such that $p(F)$ is compact will be called the minimal polynomial of $F$. We denote by $\Xi(X)$ the subset of $P \mathscr{K}(X)$ defined by

$$
\begin{gathered}
\Xi(X):=\{F \in P \mathscr{K}(X) \text { such that the minimal polynomial of } F \\
\left.p(z)=\sum_{r=0}^{p} a_{r} z^{r} \text { satisfies } p(-1) \neq 0\right\} .
\end{gathered}
$$

We first prove the following lemma which is required in the sequel.

LEMMA 4.1. If $F \in \Xi(X)$, then $I+F \in \Phi(X)$ and $i(I+F)=0$.

Proof. Since $p(F) \in \Xi(X)(p(\cdot)$ denotes the minimal polynomial of $F)$, then $\sigma_{b}(p(F))=\{0\}$. By hypothesis $p(-1) \neq 0$, then $p(-1) \notin \sigma_{b}(p(F))$. Next, making use of the spectral mapping theorem for the Browder essential spectrum [5, Theorem 4] we conclude that $-1 \notin \sigma_{b}(F)$, that is, $-1 \in \rho_{b}(F)$. This ends the proof.

The developments below are mainly suggested by the fact that, in general, the sets $\mathscr{F}(X)$ and $\Xi(X)$ do not coincide. Indeed, if $p(z)=\left(z-\lambda_{1}\right)^{n_{1}} \cdots(z-$ $\left.\lambda_{k}\right)^{n_{k}}$ is the minimal polynomial of $F \in \Xi(X)$, then, by the structure theorem of Gilfeather [3, Theorem 1], the spectrum of $F$ consists of countably many points with $\left\{\lambda_{1}, \ldots, \lambda_{k}\right\}$ as only possible limit points and such that all but possibly $\left\{\lambda_{1}, \ldots, \lambda_{k}\right\}$ are eigenvalues with finite-dimensional generalized eigenspaces. This, together with the fact that the operators belonging to $\mathscr{F}(X)$ satisfy the Riesz-Schauder theory of compact operators (see above), implies that $\mathscr{F}(X) \neq$ $\Xi(X)$. Thus the next result improves Proposition 3.6.

Proposition 4.2. Let $(U(t))_{t \geq 0}$ be a $C^{0}$-semigroup on $X$. If

$$
\{t>0 \text { such that } U(t)-I \in \Xi(X)\} \neq \varnothing \text {, }
$$

then $(U(t))_{t \geq 0}$ can be embedded in a $C_{0}$-group on $X$.

Proof. By hypothesis, there exists $t_{0}$ such that $U\left(t_{0}\right)-I \in \Xi(X)$. Since $U\left(t_{0}\right)=I+\left[U\left(t_{0}\right)-I\right]$, the use of Lemma 4.1 implies that $U\left(t_{0}\right) \in \Phi(X)$. Now, the result follows from Theorem 2.1.

Due to some technical difficulties, we do not know whether or not Theorem 3.5 is valid for perturbations belonging to $\Xi(X)$. So, we discuss this result for a subset of $\Xi(X)$ consisting of power compact operators, that is,

$$
\mathscr{P}(X):=\left\{F \in \mathscr{L}(X) \text { such that } F^{n} \in \mathscr{K}(X) \text { for some integer } n \geq 1\right\} .
$$


Our principal motivation here rely on the fact that, for some classes of Banach spaces, we have $\mathscr{F}(X) \subseteq \mathscr{P}(X)$. In particular, if $X$ is isomorphic to an $L_{p}$ space with $1 \leq p \leq \infty$ or to $C(\Omega)$ where $\Omega$ is a metric compact Hausdorff space, then $\mathscr{S}(X)=\mathscr{F}(X)$ (cf. (3.3)). Moreover, by [10, Theorem 1], we have $\mathscr{S}(X) \mathscr{Y}(X) \subseteq \mathscr{K}(X)$. These conclusions are also valid if $X$ is an $l_{p}$ space with $1 \leq p<\infty$ and $c_{0}$ [6]. Note also that if $X$ has the Dunford-Pettis property (a Banach space $X$ is said to have the Dunford-Pettis property if for every Banach space $Y$ every weakly compact operator $T: X \rightarrow Y$ takes weakly compact sets in $X$ into relatively norm compact sets of $Y$ ), then $\mathscr{W}(X)^{\mathscr{W}}(X) \subseteq \mathscr{K}(X)$ where $\mathscr{W}(X)$ stands for the set of weakly compact operators. However, although the inclusion $\mathscr{P}(X) \subseteq \mathscr{R}(X)$ is valid for arbitrary Banach spaces (use the Ruston characterization of Riesz operators [1]), in general, we have $\mathscr{P}(X) \neq \mathscr{F}(X)$. In the light of these observations, we project to extend Theorem 3.5 to semigroups $(U(t))_{t \geq 0}$ for which there exists $t_{0}>0$ such that $U\left(t_{0}\right)-I \in \mathscr{P}(X)$. Evidently, since $\mathscr{P}(X) \subseteq \Xi(X)$, Proposition 4.2 holds also true for power compact perturbations. More precisely, we have the following theorem.

THEOREM 4.3. Let $(U(t))_{t \geq 0}$ be a $C_{0}$-semigroup on $X$ with type $\omega$ and let $A$ denote its infinitesimal generator. Define the set 0 by

$$
\mathcal{O}=\{t \geq 0 \text { such that } U(t)-I \in \mathscr{P}(X)\}
$$

Then, the following items are equivalent:

(i) $\mathrm{O}=] 0,+\infty[$;

(ii) $A \in \mathscr{P}(X)$;

(iii) $[\lambda R(\lambda, A)-I] \in \mathscr{P}(X)$ for some (in fact for all) $\lambda>\omega$.

Proof. We try to imitate the procedure in the proof of Theorem 3.5. Let us first observe that if $U(t)-I \in \mathscr{P}(X)$, then there exists $m \geq 1$ such that $(U(t)-I)^{m} \in \mathscr{K}(X)$. Using the spectral mapping theorem (see, e.g., [15, page $227])$, one sees that that spectrum of $U(t)-I$ is either finite or a countable set accumulating only at zero. Moreover,

$$
\sigma(U(t)-I)=\sigma(U(t))-1
$$

This means that, apart possibly from the point $1, \sigma(U(t))=\left\{e^{\eta t}: \eta \in\right.$ $P \sigma(A)\}(P \sigma(A)$ stands for the point spectrum of $A)$ and, for any $\varepsilon>0$, the set $\{\lambda \in \sigma(U(t)):|\lambda-1|>\varepsilon\}$ is finite for all $t>0$. Then arguing as in the proof of [2, Theorem 2], we conclude that (i) implies that $A \in \mathscr{L}(X)$. Furthermore, similar arguments as in the proof of Theorem $3.5[(\mathrm{i}) \Rightarrow(\mathrm{ii})]$ imply that

$$
A=\left[\int_{0}^{t} U(s) d s\right]^{-1}(U(t)-I)=(U(t)-I)\left[\int_{0}^{t} U(s) d s\right]^{-1}
$$

which leads to $A \in \mathscr{P}(X)$. 
The remainder of the proof is verbatim that of Theorem 3.5. It suffices to use the fact that $U(t)-I$ and $\left[\int_{0}^{t} U(s) d s\right]^{-1}$ (resp., $A$ and $R(\lambda, A)$ ) commute.

We close this section by noticing that Proposition 3.6 is also valid for power compact perturbations. The proof uses Theorem 4.3.

\section{REFERENCES}

[1] S. R. Caradus, W. E. Pfaffenberger, and B. Yood, Calkin Algebras and Algebras of Operators on Banach Spaces, Lecture Notes in Pure and Applied Mathematics, vol. 9, Marcel Dekker, New York, 1974.

[2] J. R. Cuthbert, On semi-groups such that $T_{t}-I$ is compact for some $t>0, \mathrm{Z}$. Wahrscheinlichkeitstheorie und Verw. Gebiete 18 (1971), 9-16.

[3] F. Gilfeather, The structure and asymptotic behavior of polynomially compact operators, Proc. Amer. Math. Soc. 25 (1970), 127-134.

[4] I. C. Gohberg, A. S. Markus, and I. A. Fel'dman, Normally solvable operators and ideals associated with them, Amer. Math. Soc. Trans. Ser. 261 (1967), 6384.

[5] B. Gramsch and D. Lay, Spectral mapping theorems for essential spectra, Math. Ann. 192 (1971), 17-32.

[6] R. H. Herman, On the uniqueness of the ideals of compact and strictly singular operators, Studia Math. 29 (1968), 161-165.

[7] E. Hille and R. S. Phillips, Functional Analysis and Semi-Groups, American Mathematical Society Colloquium Publications, vol. 31, American Mathematical Society, Rhode Island, 1957.

[8] T. Kato, Perturbation theory for nullity, deficiency and other quantities of linear operators, J. Analyse Math. 6 (1958), 261-322.

[9] K. Latrach and A. Dehici, Relatively strictly singular perturbations, essential spectra, and application to transport operators, J. Math. Anal. Appl. 252 (2000), no. 2, 767-789.

[10] K. Latrach and A. Jeribi, Some results on Fredholm operators, essential spectra, and application, J. Math. Anal. Appl. 225 (1998), no. 2, 461-485.

[11] M. Schechter, On the essential spectrum of an arbitrary operator. I, J. Math. Anal. Appl. 13 (1966), 205-215.

[12] _ Riesz operators and Fredholm perturbations, Bull. Amer. Math. Soc. 74 (1968), 1139-1144.

[13] J. N. Vladimirskiŭ, Strictly cosingular operators, Soviet Math. Dokl. 8 (1967), 739740.

[14] R. J. Whitley, Strictly singular operators and their conjugates, Trans. Amer. Math. Soc. 113 (1964), 252-261.

[15] K. Yosida, Functional Analysis, 6th ed., Grundlehren der Mathematischen Wissenschaften, vol. 123, Springer-Verlag, Berlin, 1980.

Khalid Latrach: Département de Mathématiques, Université de Corse, 20250 Corte, France

E-mail address: 1atrach@univ-corse.fr

Abdelkader Dehici: Département des Sciences Exactes (Branche Mathématiques), Université Du 8 Mai 1945, BP 401, 24000 Guelma, Algérie 


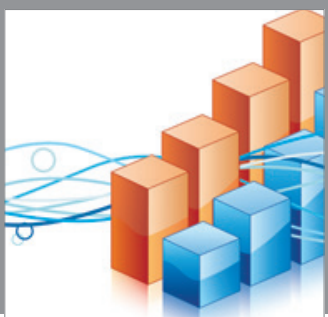

Advances in

Operations Research

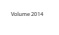

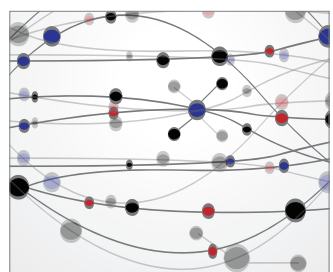

\section{The Scientific} World Journal
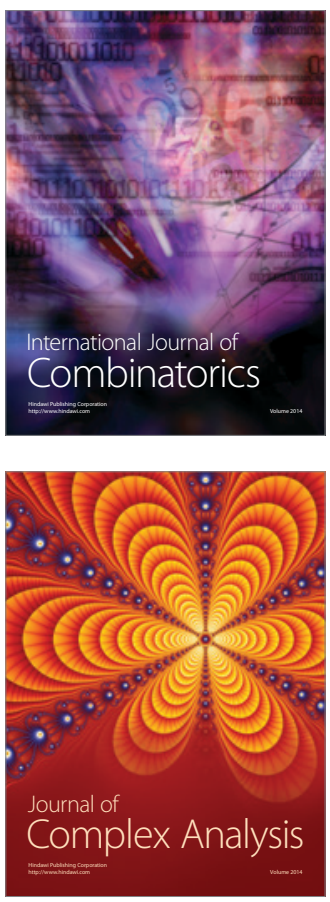

International Journal of

Mathematics and

Mathematical

Sciences
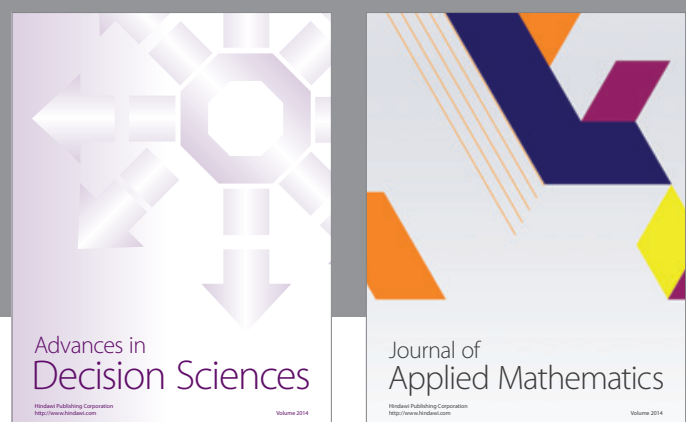

Journal of

Applied Mathematics
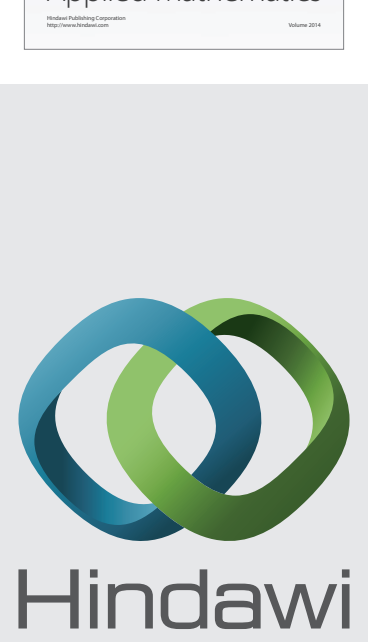

Submit your manuscripts at http://www.hindawi.com
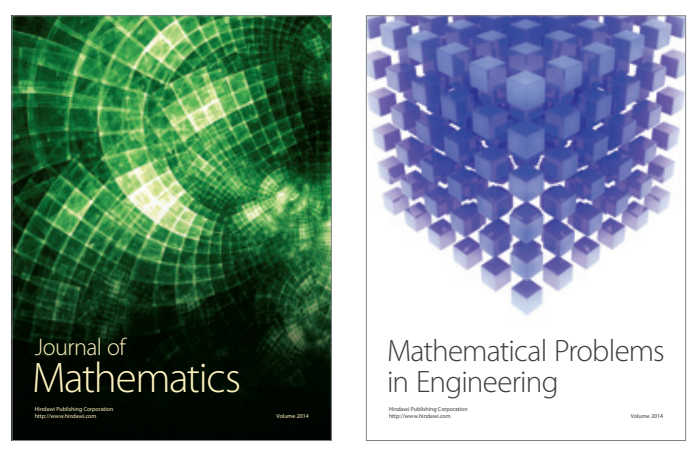

Mathematical Problems in Engineering
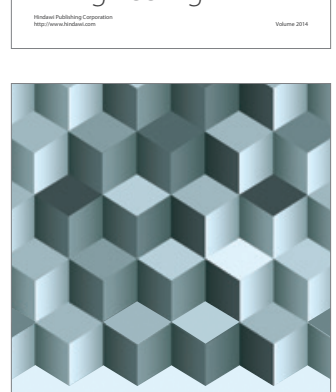

Journal of

Function Spaces
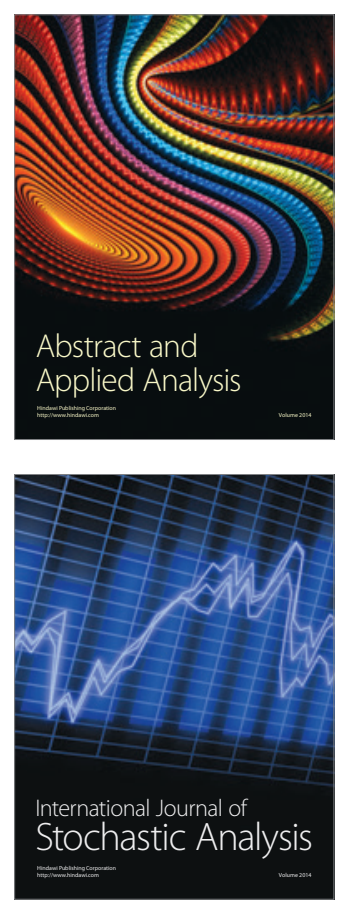

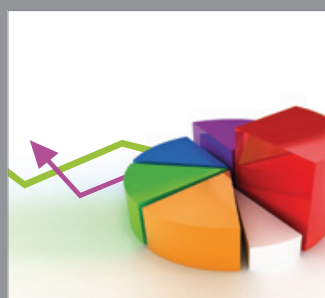

ournal of

Probability and Statistics

Promensencen
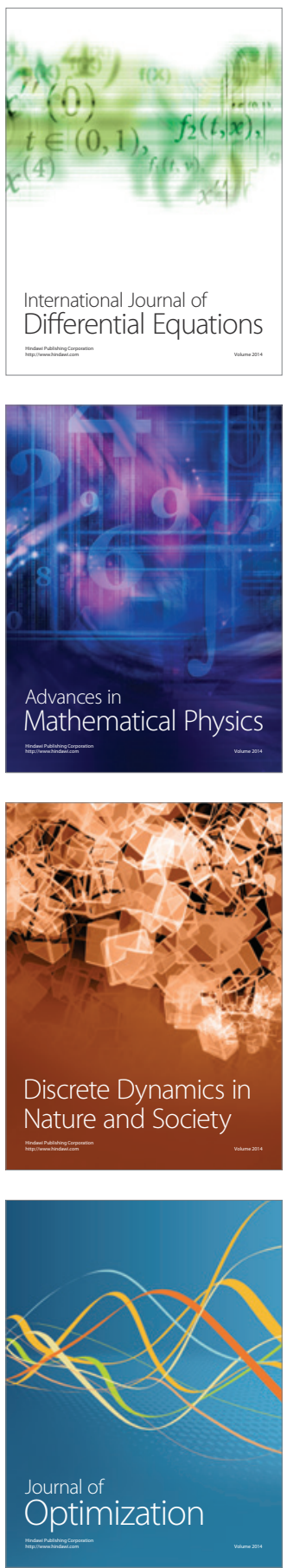